\title{
Design of Fuzzy Cognitive Model of Mutual Influence and Connectivity of Innovative Technologies
}

\author{
Sergey Gorbachev, Tatyana Abramova \\ National Research Tomsk State University, Tomsk, Russia \\ E-mail:spp03@sibmail.com
}

\begin{abstract}
The article is devoted to research and development of fuzzy cognitive model of mutual influence and connectivity of innovative technologies on the basis of synthesis of interdisciplinary research strategies foresight methods based on logically transparent mechanisms of interpretation of the solution, expert weighting methods and neutrosophic cognitive maps. Neutrosophic cognitive map of mutual influence and connectivity of innovative technologies is constructed on the basis of data on the nature and intensity of these interactions, allowing to calculate the degree of influence of one or several concepts (technologies), taking into account their state, on the target indicator, and also calculate the fixed state of the concept system. The results of experimental verification and the multiplicative effect of the developed model on the data of expert surveys are described, under conditions of transition to the 6th technological order.
\end{abstract}

Keywords: innovative technologies; foresight; experts; polls; fuzzy cognitive model; forecast

\section{Introduction}

The problem of efficiency of scientific and technological developments during the change of technological orders (TO) was first staged in economic science in the early XX century. The theory of economic cycles developed by the russian scientist N.D. Kondratiev [1], has become an integral part of the theory of innovation developed by the american economist Schumpeter. The basic postulates of his theory are as follows:

1) innovation occurs in leaps and bounds - over time, this leads to economic cycles;

2) economic cycles determined by technological innovation or innovation (figure 1).

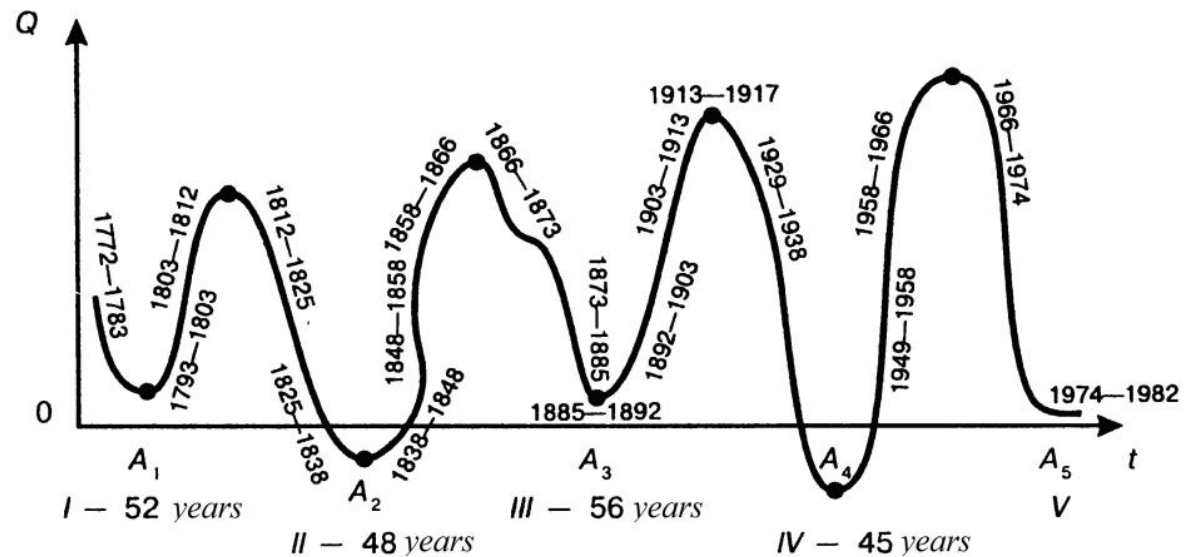

Figure 1. Structure of economic cycles and technological orders

The researchers note one important feature of TO change: the origin of all innovation starts much earlier in their mass adoption. I.e., their discovery and invention happens in a technological way, and the mass use in the following.

Among the well-known russian economists especially are the followers of N.D. Kondratiev - D.S. Lvov and S. Glazyev, which is scientifically processed and adapted the concept of "technological order" [2]. According to them, a certain way of life (or a long cycle industry, in western terminology) begins when producers have at their disposal a new set of technological innovations. For example, the 5th cycle of the industry in the late twentieth century was associated with the development of computer technology and software, genetic engineering, digital networks and communication.

In a broader theoretical framework for the formalization of techno-economic processes are closely connected with questions of the adequacy of global models. Despite some success D. Forrester and D. Meadows, according 
to experts, the macro model of world dynamics, described by the authors 1970-x years, was too mechanistic: it was not taken into account the regional structure of the object, social, political factors, there were differences with the theories of economic growth were not taken into account the adaptive capacity of the world system with social, scientific and technological progress [3].

We believe that the main perspective and the novelty of the development of mathematical methods of feasibility analysis lies in the use and development of expert (foresight methods) in combination with intelligent methods of analysis and forecasting. In [4,5] for the first time describes the neuro-fuzzy predictive model of the trajectory of world economic and technological development (TED), allowing to measure the level and pace of TED countries relative to the reference trajectory.

In continuation of these works, the aim of this study is:

1) development of an extended conceptual model of foresight methods;

2) development of a fuzzy cognitive model of mutual influence and connectivity of innovative technologies based on foresight methods;

3) experimental verification of the developed model on the example of innovative technologies.

\section{Design of fuzzy cognitive model of mutual influence and connectivity of innovative technologies}

\subsection{An extended conceptual model of foresight methods}

At present, a system of expert evaluation methods known as Foresight is widely known to identify priority areas of science, technological breakthroughs, strategic prospects for innovative development.

One of the basic methods of Foresight is the Delphi method, which is a multi-level expert survey with feedback [4]. Researchers note the continuous development of the system of Forsyte methods - over the past 20 years there is a great practical experience of their application. At present, there are several dozen methods that can be divided into quantitative and qualitative methods.

Quantitative methods include analysis of mutual influence, extrapolation, modeling, analysis and forecast of indicators, etc. Qualitative methods include interviews, literature reviews, morphological analysis, matching trees, scenarios, role plays, etc.

A completely specific set of methods for different projects may differ depending on many factors.

The results of the analysis of publications testify to the emerging tendency of a constant complication of the system of methods used. As for mega-projects devoted to the choice of technological priorities at the national level, here, in our opinion, new intellectual approaches are required to ensure objective assessments based not only on the quantitative analysis of empirical data (statistical indicators, patent statistics, bibliometric information), but and qualitative interpretation, with obtaining logically transparent mechanisms for explaining the solution. In this connection, the author of the article for the first time proposed a modified concept of foresight methods based on the addition of another vertex in the "foresight rhombus" of I. Miles and R. Popper [6] - "transparency" that transforms the original triangle into a pentagon (Figure 2).

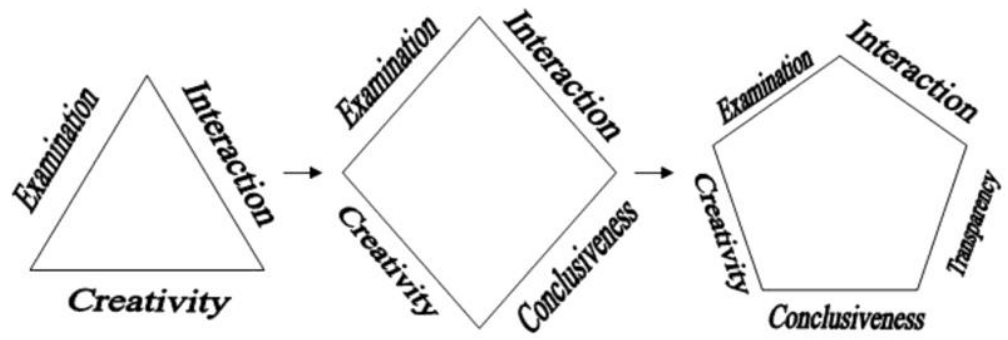

Figure 2. An extended conceptual model of foresight methods

Thus, foresight methods can be grouped into five key categories:

1) Expertise: for verification of the results obtained based on the analysis of primary sources, it is necessary to discuss the development trends of the subject area with key experts in this field, representing science, business, public organizations;

2) Creativity: to anticipate the unexpected turns of the future development, it is necessary to introduce elements of creativity in analysis. A typical example of the creative methods are wild cards ("random factors", "Joker") and weak signals ("weak signals"). Thus, the essence of the method of wild cards is that on the basis of various kinds of information (scientific and journalistic articles, interviews with experts, etc.) identifies events that have a low probability of occurrence, but are able to exert great influence on the further course of 
development. One of the salient examples of wild cards is the accident at the nuclear power plant "Fukushima", which forced the international community to take a new look at the prospects of nuclear power development;

3) Interaction: in the process of foresight needs to involve as many stakeholders, including representatives of science, business, and government, providing them with an ongoing dialogue regarding future development. If the subject of the research affects the wider society, much attention should be paid to informing the public about the progress of the foresight and its main conclusions. This is achieved by conducting different workshops, panels of citizens, etc.;

4) Conclusiveness: the reliability of results of foresight must be based on previously published works and to study the best international practices in the subject area;

5) Transparency (transparency): quantitative results should be accompanied by qualitative interpretations that are logically transparent mechanisms explain the solution using Data Mining methods (identification of knowledge from data).

\subsection{Concept of expert weighing}

According to S. Glazyev, currently it can be to observe the emergence of a new technological order as a certain set of conjugate technology basic industries, at roughly the same level of technological development [2]. Therefore, an important condition for the formation of a new technological order is rapport and interaction as a basis for the formation of load-bearing sectors and clusters of economy of the new technological order.

In the course of foresight research [6] experts were asked on a 10-point scale to assess the degree of interaction between technologies from the point of view of influence on the process of conjugation and formation of supporting industries and clusters the new economy TO (assessment of the impact of technology X on technology $\mathrm{Y}$, in terms of their interactions for the formation of supporting industries may be different from the assessment of the impact of technology $\mathrm{Y}$ on technology $\mathrm{X}$ ). The answer was formulated in the form of a triplet (amin, a, amax).

Based on the source of information, experience and intuition of the experts often are confident enough to quantify the bounds (intervals) of possible (allowed) values of the parameters and their most probable (preferred) values. For example, experts can describe primary data using triangular fuzzy numbers with the following membership function $\mu_{A}(x)$ of fuzzy set $A$ (figure 3 ):

$$
\mu_{A}(x)= \begin{cases}0, & x \leq a \\ \frac{x-a}{b-a}, & a \leq x \leq b \\ \frac{c-x}{c-b}, & b \leq x \leq c \\ 0, & c \leq x\end{cases}
$$

The following formula simulates the position of the expert: "parameter $A$ is approximately equal to $b$ and is clearly part of the segment $[a, c]$ ".

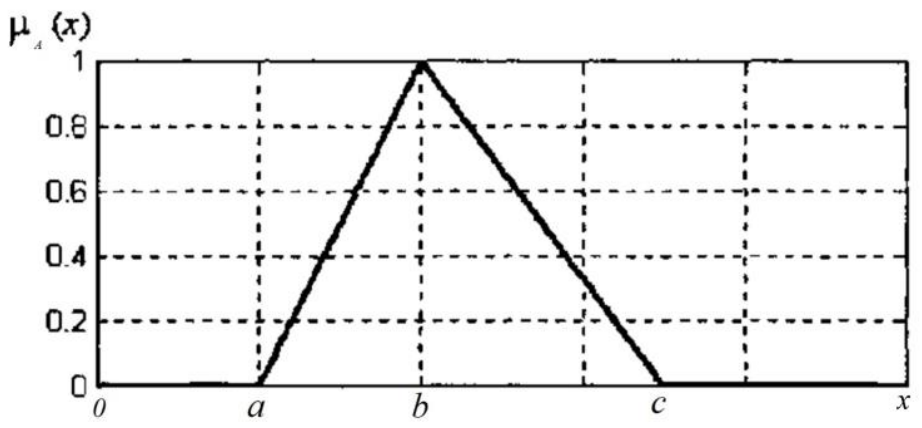

Figure 3 Triangular membership function

\subsection{Neutrosophic cognitive maps}

For research and development of a complex system interacting with other factors (concepts) on the basis of data on the nature and intensity of these interactions in cognitive studies in recent years, often uses fuzzy cognitive maps (FCM) [7], first introduced in the scientific use by B. Kosko in 1986. The contrast to the classical cognitive maps lies in the fact that the FCM represent a fuzzy directed graph whose nodes are fuzzy sets. Directed edges of the graph not only represent the causal relationships between concepts, but also determine the degree of influence (weight) of linked concepts in the interval [0;1]. 
Thus, the FCM defined 2 relationships between factors:

1) the absence of relations;

2) having relations with a certain degree of influence.

In the publication [8] indicates a third option of interaction between elements of the system - uncertainty. Obviously, it is applicable in cases when it is impossible to determine the mutual influence between concepts, but also to deny its existence is also not warranted. In this case, edges corresponding to the relations of uncertainty, denoted by the dashed line, the values of intensity I. Such as cognitive maps got the name neutrosophic cognitive maps (NCM). The results show that compared with fuzzy cognitive maps the neutrosophic cognitive maps allow more accurate modeling of complex systems in real conditions, in addition:

1) there is no need for prior specification of concepts and relations of influence;

2) there is a possibility of visual presentation of the simulated subject area within the system;

3) NCM are constructive, visibility and relative ease of interpretation with their help, the cause-and-effect relationships (relations) between concepts;

4) there is integration with the methods of assessing the results of the analysis.

Part of the relationship between factors can be determined using statistical methods [9]. At the same time using NCM can supplement the other concepts and relationships, using expert methods. In other words, in the conditions of incompleteness of the statistical data to generate accurate output you have the option to supplement the primary data base expertise - this procedure allows you to increase the base to generate a potentially more reliable conclusion.

The general approach for the formation of NCM include:

1) formation of a list of factors of the analyzed system, usually relevant expert issues foresight studies;

2) establishment of relationships between them, on the basis of expert assessments.

To more correctly describe the methods of neutrosophic cognitive modeling, it is necessary to bring the base definitions [8].

Definition 1.

NCM with edges weights taking values $\{[-1,1], \mathrm{I}\}$ is called a complex NCM.

Definition 2.

Let $\mathrm{C}_{1}, \mathrm{C}_{2}, \ldots, \mathrm{C}_{\mathrm{n}}$ are nodes of NCM, then neutrosophic matrix of this map is defined as $N(E)=\left(e_{i j}\right)$, where $e_{i j}$ is the weights of directed edges $C_{i} C_{j}$, takes values $e_{i j} \in\{[-1,1], I\}$. In this case, $N(E)$ is called neutrosophic adjacency matrix.

Definition 3.

Let $A=\left(a_{1}, a_{2}, a_{3}, \ldots, a_{n}\right)$, where $a_{i} \in\{0,1, I\}$, then $A$ is called neutrosophic the instantaneous state vector, denoting on, off or indeterminate state of the node at the moment, in other words:

$a_{i}=0$ if the node is off (has no effect),

$a_{i}=1$ if the node is enabled (it is single exposure),

$a_{i}=I$, if the node is not defined (impact not specified).

Definition 4.

Natcc is called cyclic if its edges form a directed cycle. If the causality runs through the entire cycle, NCM called a dynamic system.

Definition 5.

Let the nodes $C_{1}, C_{2}, \ldots, C_{n}$ form a cycle, then if the node $C_{i}$ is enabled and causality, going through the cycle, returns back to $C_{i}$, then this means that the dynamical system moving in a circle. If this is true for any node $C_{i}$ for $i=1,2, \ldots, n$, then this situation is called equilibrium position of a dynamical system or a hidden pattern of NCM.

The state vector, which is the equilibrium position, called the fixed point.

Using NCM possible to establish the degree of influence of a particular concept (or group of concepts) on other concepts of the system is influenced not influenced and targeted [10,11]. Thus there is a possibility to predict the degree of implementation of the target. Thus, the so-called multiplicative effect can be achieved. It is characterized by the increasing influence by a certain node in the graph causes the increase of influence to the next node in the causal chain.

\subsection{Experimental results and discussion}

Based on the conducted foresight research experts evaluated including the degree of interaction of innovative technologies in conditions of transition to the 6th technological order, from the point of view of influence on the process of conjugation and formation of the load-bearing sectors and clusters of economy of the new technological order.

In Figure 4 shows Neutrosophic cognitive map of mutual influence and connectivity of innovative technologies, generated by the results of foresight studies (numbering corresponds to their technology list in the questionnaire). 


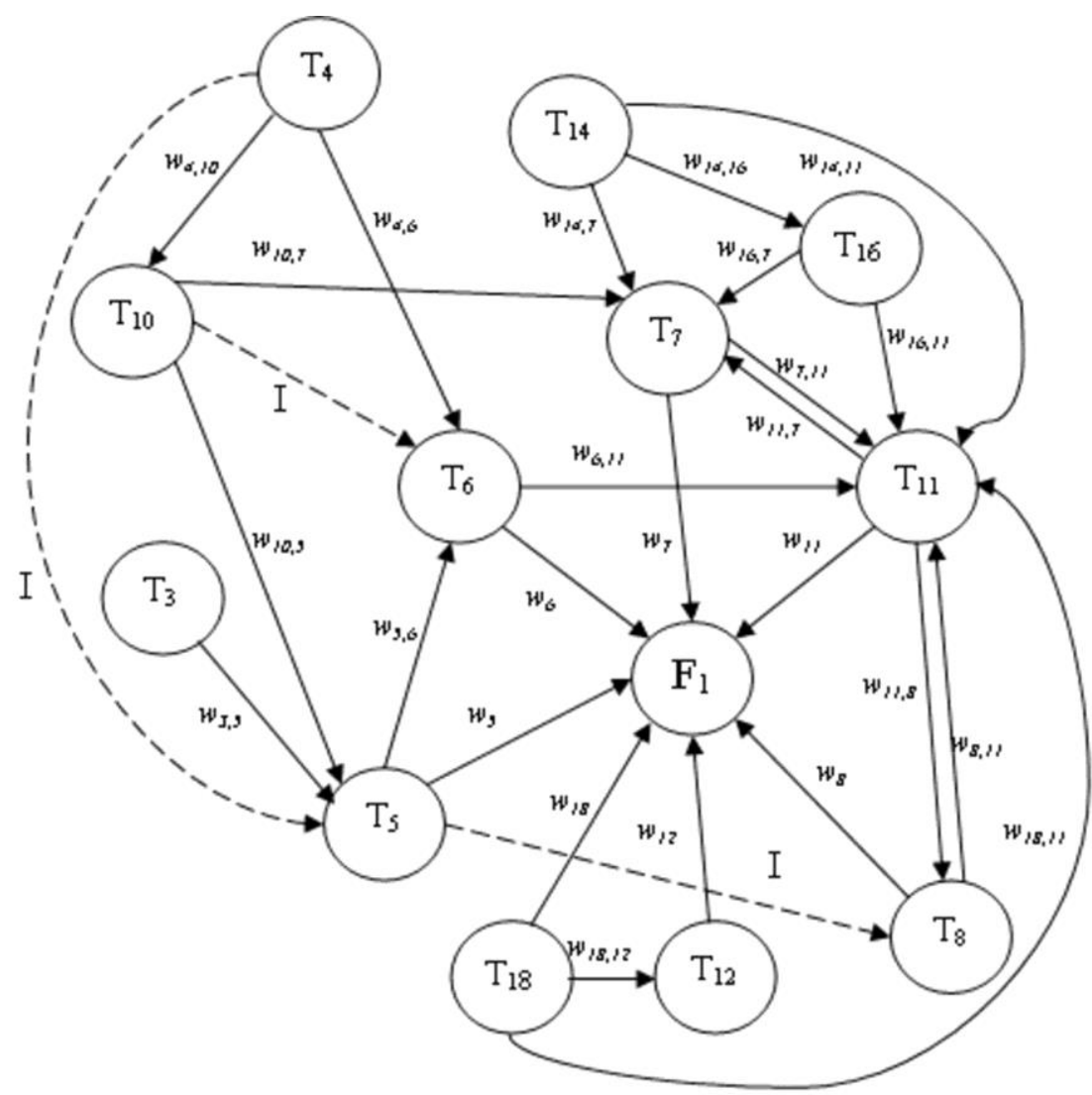

Figure 4. Neutrosophic cognitive map of mutual influence and connectivity of innovative technologies

The adjacency matrix obtained from the Neutrosophic cognitive map has the form:

$\begin{array}{ccccccccccccc}0 & 0 & w_{3,5} & 0 & 0 & 0 & 0 & 0 & 0 & 0 & 0 & 0 & 0 \\ 0 & 0 & I & w_{4,6} & 0 & 0 & w_{4,10} & 0 & 0 & 0 & 0 & 0 & 0 \\ 0 & 0 & 0 & w_{5,6} & 0 & I & 0 & 0 & 0 & 0 & 0 & 0 & w_{5} \\ 0 & 0 & 0 & 0 & 0 & 0 & 0 & w_{6,11} & 0 & 0 & 0 & 0 & w_{6} \\ 0 & 0 & 0 & 0 & 0 & 0 & 0 & w_{7,11} & 0 & 0 & 0 & 0 & w_{7} \\ 0 & 0 & 0 & 0 & 0 & 0 & 0 & w_{8,11} & 0 & 0 & 0 & 0 & w_{8} \\ 0 & 0 & w_{10,5} & I & w_{10,7} & 0 & 0 & 0 & 0 & 0 & 0 & 0 & 0 \\ 0 & 0 & 0 & 0 & w_{11,7} & w_{11,8} & 0 & 0 & 0 & 0 & 0 & 0 & w_{11} \\ 0 & 0 & 0 & 0 & 0 & 0 & 0 & 0 & 0 & 0 & 0 & 0 & w_{12} \\ 0 & 0 & 0 & 0 & w_{14,7} & 0 & 0 & w_{14,11} & 0 & 0 & w_{14,16} & 0 & 0 \\ 0 & 0 & 0 & 0 & w_{16,7} & 0 & 0 & w_{16,11} & 0 & 0 & 0 & 0 & 0 \\ 0 & 0 & 0 & 0 & 0 & 0 & 0 & w_{18,11} & w_{18,12} & 0 & 0 & 0 & w_{18} \\ 0 & 0 & 0 & 0 & 0 & 0 & 0 & 0 & 0 & 0 & 0 & 0 & 0\end{array}$

The purpose of the analysis: using of NCM will calculate the degree of exposure to one or more of the concepts (technologies), taking into account their condition on the target.

Consider the impact on the system, for example, the concept of the $T_{5}$. Based on definition, authors show how

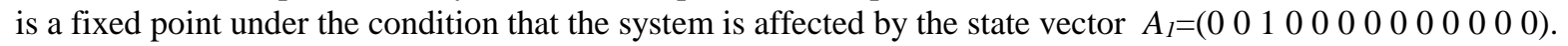

First multiply the vector $A_{l}$ on the Neutrosophic adjacency matrix of $W$, and the result will build a new state vector. Then multiply the obtained in the previous stage vector $A_{2}$ on the Neutrosophic adjacency matrix $W$, preadding $l$ to the third component of vector (this is necessary in order to account for the initial single exposure). Next to the received result of the multiplication in a similar way write the state vector. Iterative procedure is conducted until the state vector at step $n$ will be equal to the state vector at step $n-1$. This means that the result of the iterative procedure we managed to reach the fixed point:

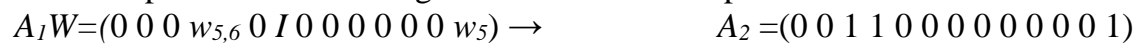

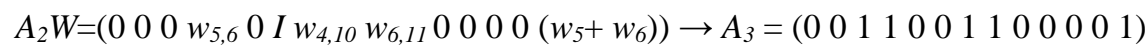




$$
\begin{aligned}
& A_{3} W=\left(00 w_{10,5}\left(w_{5,6}+I\right)\left(w_{10,75}+w_{11,7}\right)\left(I+w_{11,8}\right) 0 w_{6,11} 0000\left(w_{5}+w_{6}+w_{11}\right)\right) \rightarrow
\end{aligned}
$$

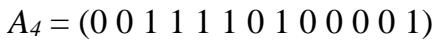

$$
\begin{aligned}
& A_{4} W=\left(000 w_{5,65} w_{11,7}\left(I+w_{11,8}\right) 0\left(w_{6,11}+w_{7,11+} w_{8,11}\right) 0000\left(w_{5}+w_{6}+w_{7}+w_{8}+w_{11}\right)\right) \rightarrow
\end{aligned}
$$

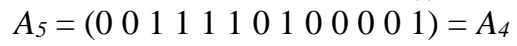

In this case a fixed point is reached in 4 iterations. The value $A_{4} W$ represents the system state vector, each element of which describes the impact applied to the corresponding concept in the result of a single impact $T_{5}$.

A special case of the simultaneous action of several of the concepts $T_{5}, T_{7}, T_{8}$, ie the effect of the vector $B_{1}=(0$ $01011000000000)$ :

$$
\begin{aligned}
& B_{1} \mathrm{~W}=\left(000 w_{5,6} 0 \text { I } 0\left(w_{7,11}+w_{8,11}\right) 0000\left(w_{5}+w_{7}+w_{8}\right)\right) \rightarrow
\end{aligned}
$$

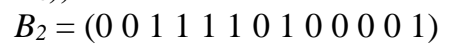

$$
\begin{aligned}
& B_{2} \mathrm{~W}==\left(00 w_{10,5} w_{5,6} w_{11,7}\left(I+w_{11,8}\right) 0\left(w_{6,11}+w_{7,11}+w_{8,11}\right) 0000\left(w_{5}+w_{6}+w_{7}+w_{8}\right)\right) \rightarrow
\end{aligned}
$$

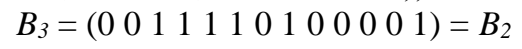

Analyzing the obtained results, it can be concluded that the change effect on the target depends on what concepts have been impacted.

The combination of neutrosophic cognitive modeling with models of neuro-fuzzy inference provides the opportunity to carry out a deep analysis of semi-structured problems. Another advantage is the ability to enter, based on expert assessment, additional primary data, which in certain cases may lead to an increase in the reliability of the conclusions to decision-makers.

\section{Conslutions}

The use of fuzzy cognitive maps as a tool for modeling semi-structured systems allows to clearly and flexibly to represent the analyzed system, as well as easy to interpret causal relationships between concepts.

The result of combining neutrosophic cognitive modeling with the results of neuro-fuzzy conclusion is the author obtained additional intelligent foresight tool, providing logical transparency of the forecasting studies. It gives the opportunity to deeply and quickly analyze scientific, technological and innovation development, taking into account many signs, their significance, mutual influence and contingency with multiplier effects, and also to carry out a situational analysis depending on the target factor. On the other hand, the methods allow for the possibility of introducing additional expert data, which in general case can increase the reliability of the conclusions.

Prospects for the development of the methods of cognitive modeling are concerned with the mathematical justification, developing algorithms for automatic construction of cognitive maps based on the data selection and adaptation of methods of training the neural network used for identification of complex dynamic systems neurofuzzy inference and control and have a practical purpose in the systems of decision support for the analysis and forecast of scientific-technological and innovative development of objects and territories.

\section{Acknowledgment}

Research performed under the program of increase of competitiveness of National research Tomsk state University among the world's leading scientific and educational centers, with the financial support of Russian Foundation for Basic Research, grant № 16-29-12858.

\section{References}

[1] Kondratiev N.D. Big cycles of conjuncture and theory of foresight. Moscow, Russia: "Economy". 2002

[2] Glazyev S.K. Theory of long-term economic development. Moscow, Russia: "VlaDar". 1993

[3] Forrester D. The global dynamics. Moscow, Russia: AST, 2003

[4] Abramova T.V., Vaganova E.V., Gorbachev S.V., Gribovsky M.V., Syryamkin V.I., Syryamkin M.V., Yakubovskaya T.V. The cognitive system of monitoring and forecast of scientific-technological development of the state. Tomsk, Russia: Publishing house of Tomsk state University. 2012.

[5] Gorbachev S.V., Abramova T.V. Neural network modeling of the trajectory of the global techno-economic development. Telecommunications, 7, 2014: p.43-49

[6] Popper R. Methodology: Common Foresight Practices \& Tools. London, UK: Edward Elgar, 2007 
[7] Kulinich A.A. Cognitive maps in decision support. Proceedings of Congress on intelligent systems and information technologies, 1, 2011: p.345-358

[8] Vasantha Kandasamy W.B., Smarandache F. Fuzzy Cognitive Maps and Neutrosophic Cognitive Maps. Xiquan: "Phoenix". 2003.

[9] Tolman E.C. Cognitive Maps in Rats and Men. Psychological Review, 55 (4), 1948: p.189-208.

[10] Taber W.R. Knowledge Processing with Fuzzy Cognitive Maps. Expert System with Applications, 2, 1991: p.83-87.

[11] Nguyen Dang Thanh; Le Hoang Son ; Mumtaz Ali Neutrosophic recommender system for medical diagnosis based on algebraic similarity measure and clustering . Proceeding of International Conference on Fuzzy Systems. 2017: p.1-6. 